\title{
Endometrial Metastasis from an Occult Colonic Primary presenting with Massive Ascites
}

\author{
${ }^{1}$ Ashok S Komaranchath, ${ }^{2}$ Sindhu Gopalakrishnan, ${ }^{3}$ Shirly John, ${ }^{4}$ Pushpa Mahadevan, ${ }^{5}$ John Mathew, ${ }^{6}$ Lijesh Kumar
}

\begin{abstract}
In most developed nations, endometrial cancer is the most common gynecological malignancy. However, the endometrium is an extremely rare site for metastasis from an extragenital site. Also, the most common presentation of such metastasis is with uterine bleeding, similar to primary endometrial cancers. We present an unusual case of a 38-year-old female who presented with abdominal pain and distension. Evaluation revealed an endometrial mass lesion with bilateral adnexal masses and massive ascites. An endometrial biopsy was done, which revealed a mucinous adenocarcinoma with signet ring cells. Immunohistochemistry showed cells positive for CK20 and CDX2 and negative for CK7, which was in favor of a colonic primary. A colonoscopy was attempted, but failed due to extrinsic compression at the rectosigmoid junction. There was no evidence of supradiaphragmatic disease. She was started on palliative chemotherapy. This case report highlights the indispensability of histological correlation and immunohistochemistry in the diagnosis of pelvic malignancies.
\end{abstract}

Keywords: Endometrial cancer, Endometrial metastasis, Signet-ring carcinoma.

How to cite this article: Komaranchath AS, Gopalakrishnan S, John S, Mahadevan P, Mathew J, Kumar L. Endometrial Metastasis from an Occult Colonic Primary presenting with Massive Ascites. J South Asian Feder Menopause Soc 2017;5(2):138-141.

Source of support: Nil

Conflict of interest: None

Date of received: 23 July 2017

Date of acceptance: 7 September 2017

Date of publication: December 2017

\footnotetext{
${ }^{1-6}$ Consultant

${ }^{1}$ Department of Medical Oncology, PVS Memorial Hospital Kochi, Kerala, India

${ }^{2,3}$ Department of Obstetrics and Gynecology, PVS Memorial Hospital, Kochi, Kerala, India

${ }^{4}$ Department of Pathology, VPS Lakeshore Global Lifecare Kochi, Kerala, India

${ }^{5}$ Department of Gastroenterology, PVS Memorial Hospital Kochi, Kerala, India

${ }^{6}$ Department of Radiology, PVS Memorial Hospital, Kochi Kerala, India

Corresponding Author: Ashok S Komaranchath, Consultant Department of Medical Oncology, PVS Memorial Hospital, Kochi Kerala, India, Phone: +914844182888, e-mail: komaranchath@ gmail.com
}

\section{INTRODUCTION}

Endometrial cancer is the most common gynecologic malignancy in the Western world, and accounts for approximately $5 \%$ of all cancers in women. There are an estimated 319,605 cases with 76,160 deaths per year in the world due to uterine cancer. ${ }^{1}$ The vast majority of these cancers are adenocarcinomas of the corpus uteri. Metastasis to the corpus uteri are rare and are from other genital primaries, usually from the ovaries. The most common locations of the primary tumor are the breast, followed by the gastrointestinal (GI) tract. Endometrial cancer is generally a disease of the elderly with the median age of diagnosis being around 60 years of age. This seems to hold true for endometrial metastases as well. The clinical presentation of primary endometrial cancers as for endometrial metastases is usually with uterine bleeding. The prognosis of endometrial metastases is unclear, but appears to be poor when the primary site is the GI tract.

\section{CASE REPORT}

A 38-year-old married premenopausal woman presented to us with complaints of progressive abdominal distension and pain of 2 months duration. She gave no history of bleeding per vaginum, malena, or any GI disturbances. She was married with no children, and gave history of treatment for infertility several years prior to the present complaints. On examination, she had massive, tense ascites and bilateral pitting pedal edema, which was very prominent on the right lower limb. Per vaginal examination revealed an irregular, indurated surface with a frozen pelvis and no free area between the pelvic walls. A contrast-enhanced computed tomography (CT) scan showed a large $6.1 \times 5.8 \mathrm{~cm}$ hypodense lesion in the uterine cavity with mixed density masses in both adnexa, $8.4 \times 4.3 \mathrm{~cm}$ on the left and $8.6 \times 4.8 \mathrm{~cm}$ on the right side (Fig. 1). There was an irregular soft tissue thickening in the sigmoid colon wall and of the omentum. There was also gross ascites, but no other evidence of metastatic disease. Our initial diagnosis was that of endometrial cancer with bilateral ovarian metastases. However, tumor markers revealed carcinoembryonic antigen (CEA) of $60.37 \mathrm{ng} / \mathrm{mL}$ and CA125 of $41.7 \mathrm{U} / \mathrm{mL}$. In view of patient distress, a diagnostic and therapeutic ascitic tap was done. The fluid was bloody, with no growth on culture and positive for 

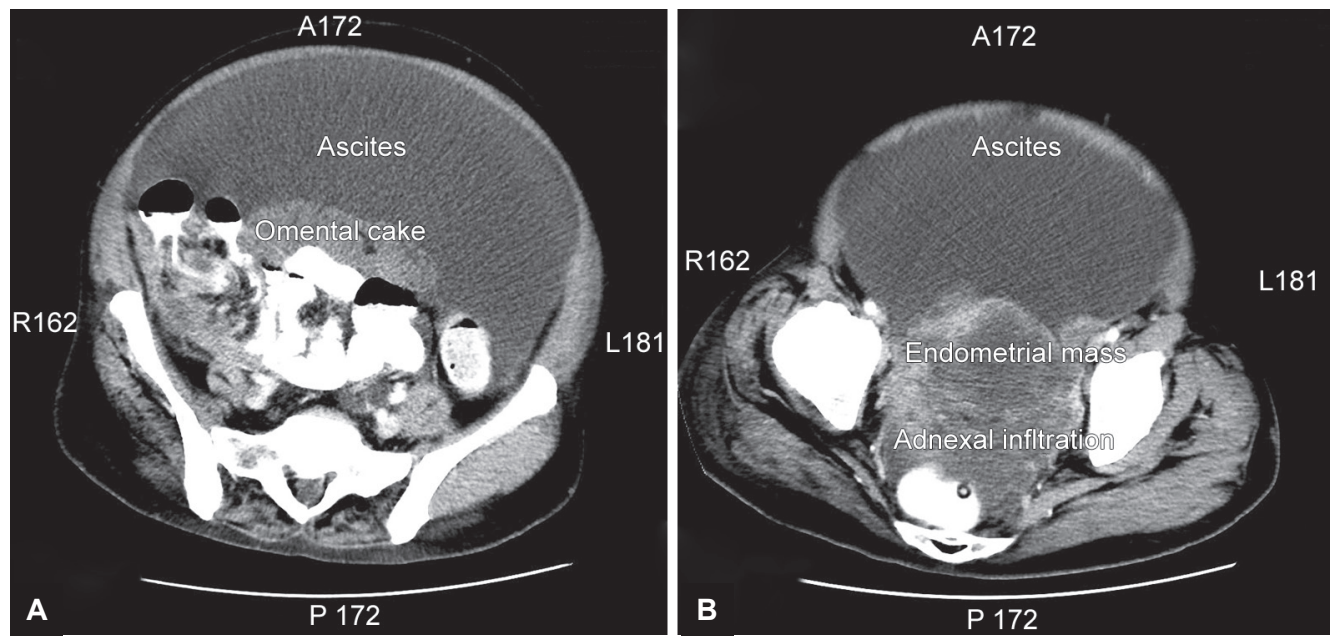

Figs $1 \mathrm{~A}$ and $\mathrm{B}$ : Computed tomography scan

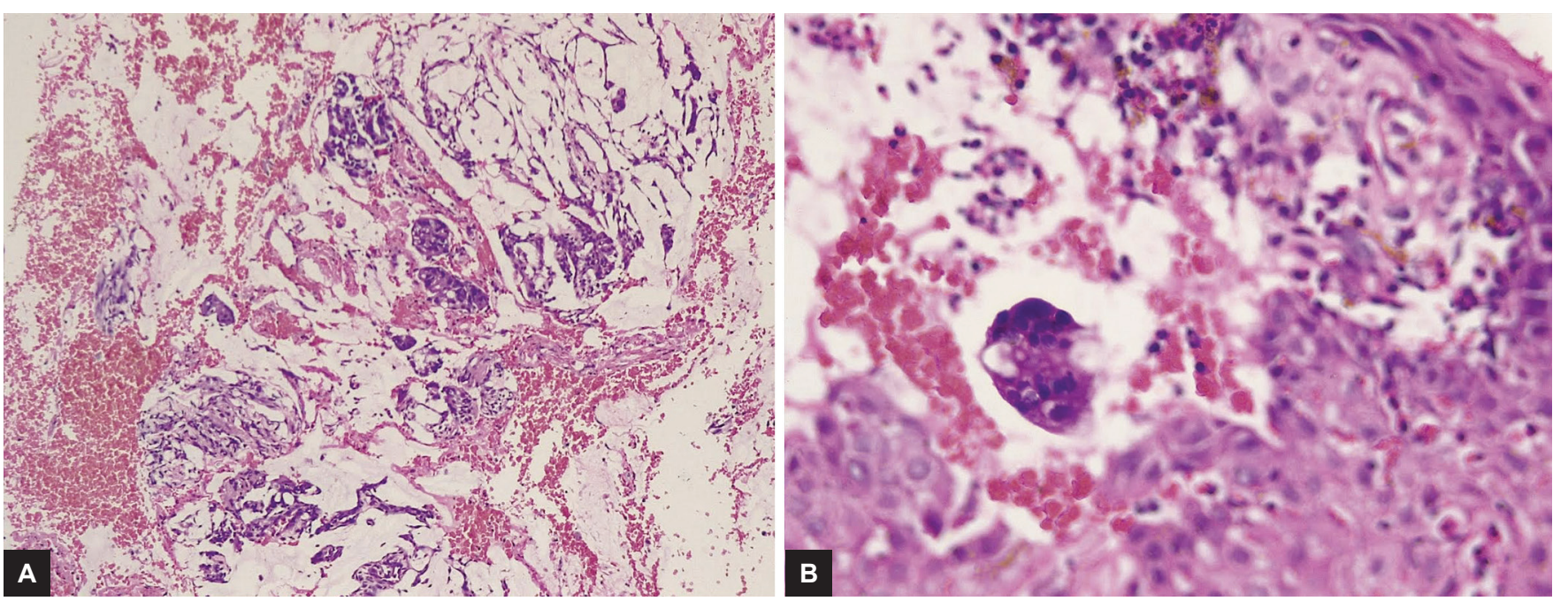

Figs 2A and B: Histopathological examination

malignant cells. An endometrial biopsy was performed and the histopathology was reported as adenocarcinoma with mucinous areas and signet ring cells (Fig. 2). As both signet ring cells and mucinous appearance are unusual for an endometrial adenocarcinoma, we went ahead with immunohistochemistry, which revealed that CK7 (Fig. 3) and CA125 were negative and CK20 and CDX2 (Fig. 4) were positive. In view of the strong possibility of a colonic primary at this stage, a colonoscopy was attempted, but the scope could not be passed beyond the rectosigmoid due to extrinsic compression. ACT scan of the thorax was done to rule out any supradiaphragmatic involvement.

We started her on chemotherapy with capecitabine $(1,000 \mathrm{mg} / \mathrm{m} 2)$ and oxaliplatin $(130 \mathrm{mg} / \mathrm{m} 2)$ regimen every 3 weeks. The endometrial biopsy specimen has been sent for rat sarcoma (RAS) mutational analysis and reports are awaited.

\section{DISCUSSION}

In most developed countries, endometrial cancer is the most common gynecological malignancy encountered.

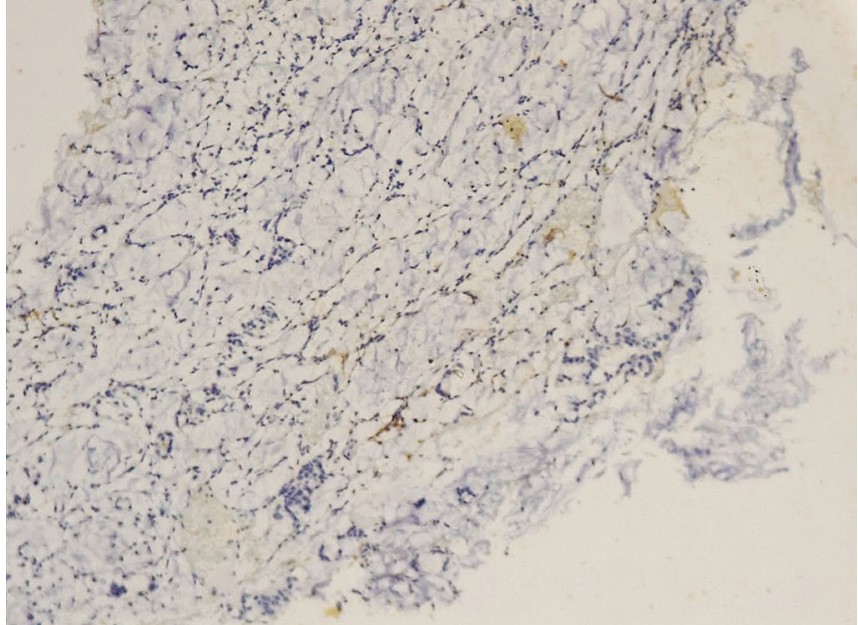

Fig. 3: CK7

Every year, there are over 300,000 cases of endometrial cancers diagnosed worldwide. The incidence in India is much less with around 12,000 cases reported each year and around 4,700 fatalities from the same. According to the Surveillance, Epidemiology, and End Results 


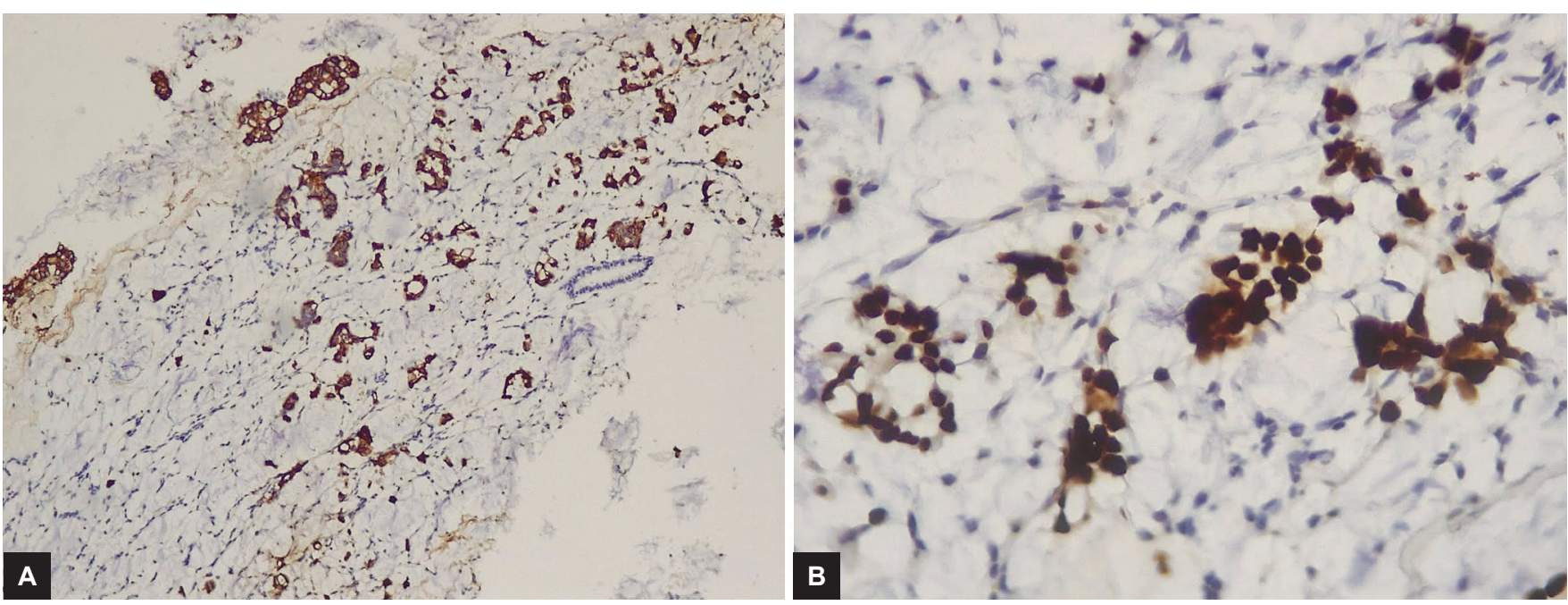

Figs $4 \mathrm{~A}$ and $\mathrm{B}$ : CK20 and CDX2

database, the median age at diagnosis for endometrial cancer is 62 years and only $7 \%$ of cases are diagnosed under the age of 44 years. $^{2}$ The most common type of endometrial cancers are adenocarcinomas followed by sarcomas and carcinosarcomas. Metastases to the genital structures are rare and usually involve the ovaries alone. The endometrium is a very rare site of metastases, especially from extragenital structures. There have been only few individual case reports and case series detailing endometrial metastasis from extragenital sites. ${ }^{3-7}$ The largest case series consisted of 63 cases published in $1982 .{ }^{8}$ The majority of primary sites in this series were in the breast $(42.9 \%)$, followed by the colon $(17.5 \%)$. Concomitant involvement of the ovaries was seen in two-thirds of the cases with endometrial metastases. The probable reason for the majority of endometrial metastases coming from the breast may have to do with the expression of hormone receptors in both these organs. The age of presentation of these patients also tends to mirror that of patients with primary endometrial cancers with a reported median age of 59.7 years. It is highly unusual for a premenopausal woman, such as our patient to present with endometrial metastases. Another unusual feature of this case was the complete lack of GI symptoms as well as a conspicuous absence of a history of uterine bleeding. A recent case report that had a similar pathology of endometrial metastasis from a colonic primary had an initial clinical presentation of dysfunctional uterine bleeding. ${ }^{9}$ Instead, our patient had a sudden onset of abdominal pain and distension with rapid progression of symptoms. There was absolutely no GI symptom nor any history of a uterine bleed.

Histologically, adenocarcinomas of the colorectum and adenocarcinomas of the corpus uteri share some morphological features, but the presence of mucinous areas and signet ring cells goes in favor of a colonic malignancy.
Immunohistochemistry has become an invaluable tool in differentiating the origin of various malignancies and was instrumental in clinching the diagnosis of a colonic primary in this case.

Although we were unable to confirm the presence of a colonic primary, the histological appearance of the endometrial biopsy along with the characteristic immunohistochemistry and with the supporting evidence of a high CEA level allowed us to conclude that the patient indeed had an endometrial metastasis with the most likely primary site being the colon. In the case series by Kumar and Hart, ${ }^{8}$ it was also noted that two-thirds of patients with metastases in the uterine corpus would have a concomitant involvement of the ovaries. This was seen in our patient as well, with large mixed-density lesions seen in both the adnexa.

We could not find any literature with regard to RAS mutational analysis from an endometrial metastatic site. However, in this case, we have sent for the exons 2, 3, and 4 mutational analyses of both KRAS and NRAS, and the results are awaited. Meanwhile, the patient has received her first cycle of chemotherapy and has tolerated the same well.

\section{CONCLUSION}

This case of endometrial and bilateral ovarian metastases from a colonic primary lesion highlights the importance of careful histopathological correlation and use of immunohistochemistry in confirming the tissue of origin in any case of metastatic disease.

\section{REFERENCES}

1. GLOBOCAN 2012 data. Available from: http:/ / globocan.iarc. $\mathrm{fr} /$ Pages/fact_sheets_population.aspx.

2. Available from: https://seer.cancer.gov/statfacts/html/corp. html. 
3. Charache H. Metastatic carcinoma in the uterus. Am J Surg 1941;53:152-157.

4. Kiaer W, Holm-Jensen S. Metastases to the uterus. Acia Pathol Microbiol Scand 1972;80:835-840.

5. Stemmermann GN. Extrapelvic carcinoma metastatic to the uterus. Am J Obstet Gynecol 1961 Dec;82:1261-1266.

6. Takeda M, Diamond SM, DeMarco M, Quinn DM. Cytologic diagnosis of malignant melanoma metastatic to the endometrium. Acta Cytol 1978 Nov-Dec;22(6):503-506.
7. Weingold $\mathrm{AB}$, Boltuch $\mathrm{SM}$. Extragenital metastases to the uterus. Am J Obstet Gynecol 1961 Dec;82:1267-1272.

8. Kumar NB, Hart WR. Metastases to the uterine corpus from extragenital cancers. A clinicopathologic study of 63 cases. Cancer 1982 Nov 15;50(10):2163-2169.

9. Zannoni GF, Vellone VG, Fadda G, Petrillo M, Scambia G. Colonic carcinoma metastatic to the endometrium: the importance of clinical history in averting misdiagnosis as a primary endometrial carcinoma. Int J Surg Pathol 2011 Dec;19(6):787-790. 